\title{
PMMA with Long-Persistent Phosphors and Its Behavior of Luminescence
}

\author{
Huang Yina (黄依娜 $)^{1}$, Jiang Xinye $(\text { 蒋莘野 })^{1}$, Cai Qiang (蔡强 $)^{2}$, Lan Ding (蓝 鼎 $)^{3}$, \\ Wan Farong (万发荣 $)^{1 *}$ \\ (1. Department of Material Physics and Chemistry, University of Science and Technology Beijing, Beijing 100083, \\ China; 2. School of Materials, University of Industry Achen, Germany; 3. National Microgravity Laboratory, \\ Institute of Mechanics, Chinese Academy of Sciences, Beijing 100080, China )
}

\begin{abstract}
A new kind of rare earth material with high efficient long-persistent phosphors, such as $\mathrm{SrAl}_{2} \mathrm{O}_{4}: \mathrm{Eu}, \mathrm{Dy}$, has been developed in recent years. The PMMA with long-persistent phosphors is typical one of applications for the phosphors . In this work, we try to probe into the affection of the manufacture process on the PMMA with long-persistent phosphors, to analyze its performance, and its luminescence behavior, especially to study the self-excitation of the PMMA with long-persistent phosphors.
\end{abstract}

Key words : long-persistent phosphors; PMMA; rare earths

CLC number : 0482.3 Document code: A Article ID: $1002-0721(2006)-0157-03$

The aluminates have a good chemical stability, anti-radiation damage, and slow aging process under the UV, X-ray and electron beam. These characters make it have a high value, so it has been investigated a lot in recent years. Most of the phosphors with matrice materials of aluminates are $\mathrm{Ba}, \mathrm{Mg}, \mathrm{Ca}$ and $\mathrm{Sr}$ aluminates alkaline earth ${ }^{[1]}$.

However, crystal materials are hard to be made into big flat in single crystals, in fact, long-persistent luminescent crystal materials are used in multi-crystals or powders shape. Such long-persistent luminescent materials, such as $\mathrm{SrAl}_{2} \mathrm{O}_{4}: \mathrm{Eu}$, Dy, need to be mixed with painting and plastics for application ${ }^{[2-7]}$, but any of them should be of nice transparency, and PMMA is the best one in transparency. So it is reasonable to combine long-persistent phosphors with PMMA ${ }^{[8]}$.

The raw material of organic glass (PMMA) is liquid $\mathrm{CH}_{2} \mathrm{C}\left(\mathrm{CH}_{3}\right) \mathrm{COOCH}_{3}$ which needs to be heated in high temperature for about $10 \mathrm{~h}$ before it turns into solid by polymerization ${ }^{[9]}$. Therefore, the long-persistent phosphors would deposit during this long time of polymerization. A new process including pre-polymerization is submitted to make the PMMA with long-persistent phosphors ${ }^{[8]}$. At the same time, the luminescence behavior of phosphors inside the PMMA is also important theoretically which will make its using more widel$y^{[10]}$. This work is trying to probe into the affection of the manufacture process on the PMMA with long-per- sistent phosphors, to analyze its mechanical performance, to investigate its luminescence behavior and especially to study the self-excitation of the PMMA with long-persistent phosphors in system.

\section{Experimental}

Liquid $\mathrm{CH}_{2} \mathrm{C}\left(\mathrm{CH}_{3}\right) \mathrm{COOCH}_{3}$ was used as the raw materials of polymerization of organic glass. The phosphors $\mathrm{SrAl}_{2} \mathrm{O}_{4}: \mathrm{Eu}$, Dy (green light), $\mathrm{Sr}_{4} \mathrm{Al}_{14} \mathrm{O}_{25}: \mathrm{Eu}$, Dy (blue light) and CaSrS (red light) were used respectively.

Put a $500 \mathrm{ml}$ plastic bottle full of $\mathrm{CH}_{2} \mathrm{C}\left(\mathrm{CH}_{3}\right)$ $\mathrm{COOCH}_{3}$ (A little steel ball and some solicitations have been put in beforehand) into a water pool and heated to $60{ }^{\circ} \mathrm{C}$, keeping the temperature for $3.5 \sim 4 \mathrm{~h}$ for pre-polymerization. When the time for the little steel ball to fall from top to bottom in the bottle reached about $15 \mathrm{~s}$, cool the bottle in cold water until the time for the little steel ball to fall from top to bottom reached about $45 \mathrm{~s}$. Add $50 \mathrm{~g}$ phosphor into the liquid and mixed round until the phosphors dispersed uniformly. Then pour the liquid into the glass models, and put the models into the water pool with the temperature of $80{ }^{\circ} \mathrm{C}$ to keep for about $15 \mathrm{~h}$. The PMMA with long-persistent phosphors may be achieved after removing the glass models .

Mechanical flexural testing machine was employed to test the mechanical property of the PMMA

Received date: $2006-07-20$; revised date: $2006-09-15$

Biography: Huang Yina (1981 - ), Female, Doctor candidate; Research field: Long-persistent phosphor

* Corresponding author (E-mail : wanfr@mater.ustb.edu.cn) 
with long-persistent phosphors. The distribution of phosphors in the organic glass was measured by metallography microscope. FLUOROMAX II Spectrophorometer was used to detect the excitation and emission spectra of the products. The decay curve of afterglow was measured by a silicon solar cell and a multimeter. CAMBRIDGE $\$ 250$ scanning electron microscopy (SEM) was employed to observe the morphology of the products and the crystal phase was confirmed with Mac Science D/MAX-RB X-ray diffraction analysis that used $\mathrm{Cu} \mathrm{K} \alpha$ radiation.

\section{Results and Discussion}

The temperature of pre-polymerization is really important. In this work, $60^{\circ} \mathrm{C}$ was used as the start temperature for pre-polymerization. The organic liquid would be removed out of water when it reaches colloid state that we need. If the temperature of pre-polymerization is under $50^{\circ} \mathrm{C}$, the rate of pre-polymerization is too slow, but if it is over $70{ }^{\circ} \mathrm{C}$, the rate is too fast to control the pre-polymerization. So the temperature of pre-polymerization should be controlled at around $60{ }^{\circ} \mathrm{C}$.

Now the pre-polymerization time of $\mathrm{CH}_{2} \mathrm{C}\left(\mathrm{CH}_{3}\right)$ $\mathrm{COOCH}_{3}$ with solicitations of weight ratio $0.75 \%$ is about $120 \mathrm{~min}$, but as the storing time of $\mathrm{CH}_{2} \mathrm{C}\left(\mathrm{CH}_{3}\right)$ $\mathrm{COOCH}_{3}$ changes, the pre-polymerization time would change as well. This is because $\mathrm{CH}_{2} \mathrm{C}\left(\mathrm{CH}_{3}\right) \mathrm{COOCH}_{3}$ is easily affected by temperature, light and some other factors.

Fig. 1 shows the cross section of pure organic glass and the PMMA with $50 \mathrm{~g} \mathrm{SrAl}_{2} \mathrm{O}_{4}: \mathrm{Eu}$, Dy powders. The pure organic glass has a uniform structure. And the phosphors' particles are distributed uniformly in organic glass. So the PMMA with long-persistent phosphors made in this way would distribute uniformly.

The PMMA with two other kinds of phosphors, such as $\mathrm{Sr}_{4} \mathrm{Al}_{14} \mathrm{O}_{25}: \mathrm{Eu}$, Dy (blue light) and CaSrS (red light) were also prepared in the same way.

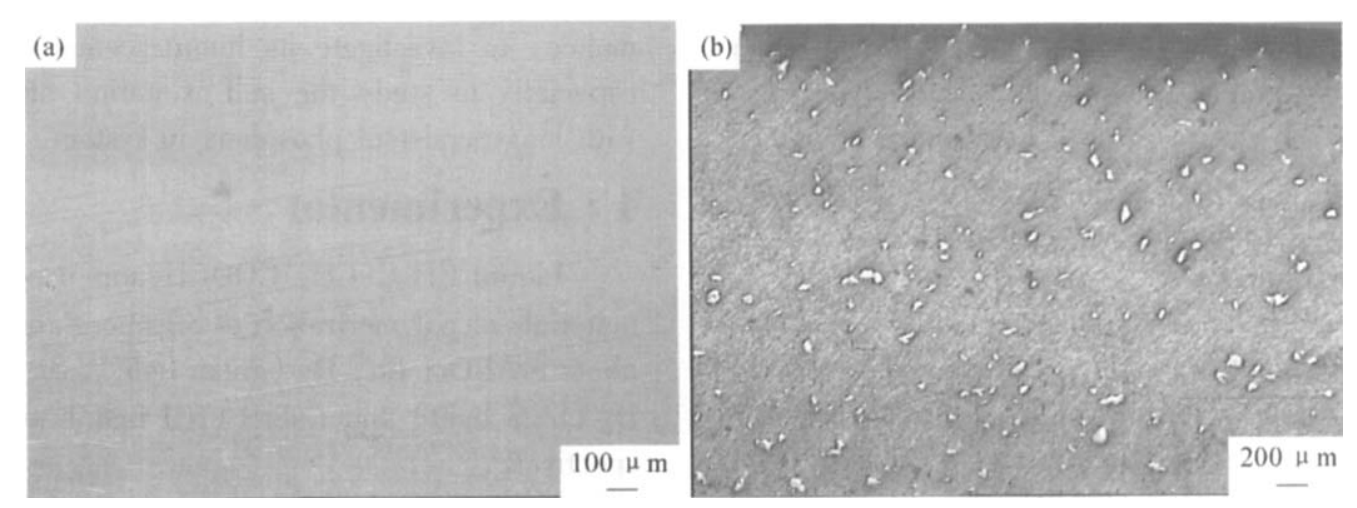

Fig. 1 Microstructure of cross section

(a) Pure PMMA; (b) PMMA with $\mathrm{SrAl}_{2} \mathrm{O}_{4}: \mathrm{Eu}, \mathrm{Dy}$

Fig. 2 shows the bending stress of the PMMA with long-persistent phosphors. The bending stress is descended as the increasing of the phosphors' content. This is because the particles in the PMMA enhance some bugs inside which makes the organic glass more fragile.

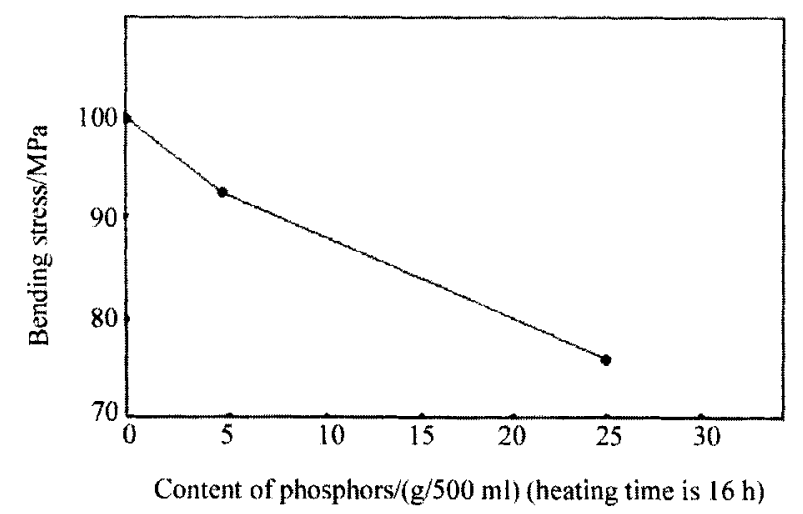

Fig.2 Bending stress curve of different contents of phosphors
Additionally, the so-called self-activation experiment of PMMA containing $\mathrm{SrAl}_{2} \mathrm{O}_{4}: \mathrm{Eu}$, Dy was carried out as well. In the experiment, half luminescent glass was excited by outside light and the other half to be covered. After a period of time in dark room, the light intensity of the excited half one became weaker, while the other un-excited half one start to sent out light gradually. At last the both half ones released the same intensity of light, then the light intensity of the whole glass decreased continuously.

Fig. 3 is the excitation and emission spectra of three phosphors with different colors. The red ones have longer light wave than the green and blue ones, while green ones have a longer wave than blue ones. So theoretically speaking, phosphors with blue light can excite green or red ones, and green one can excite red one. But from the self-activation experiment, the green one can be excited by the green one itself easier 
than the blue one. This may be due to the so-called solid syntonic absorbability, which means when the incident light has the same frequency as the systemic intrinsic frequency, the energy exchange between incident light and system is the greatest, and the system absorbs energy of the light the most strongly .

Fig. 4 is emission spectra of the PMMA containing $\mathrm{SrAl}_{2} \mathrm{O}_{4}: \mathrm{Eu}, \mathrm{Dy}$ at different time and its decay curve after activation by the UV tested by FLUOROMAX II Spectrophorometer. The emission peak would not move with time, by which means the emission peak at different time is the same. This result supported that the fluorescent light can be excited by the light

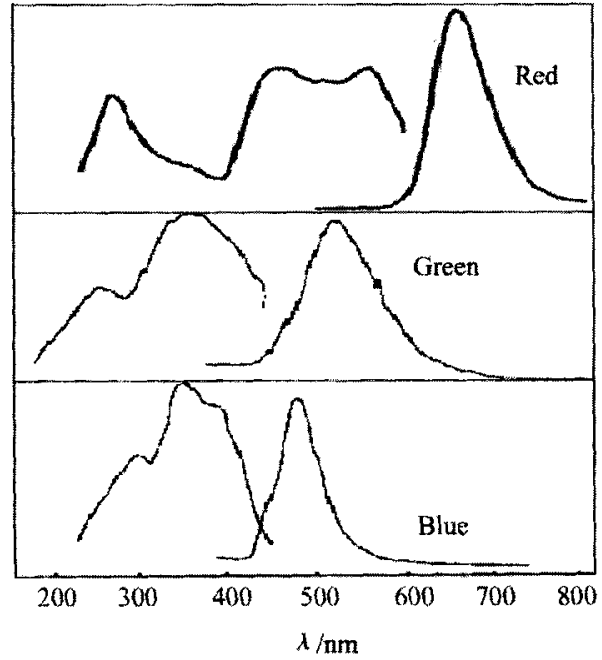

Fig.3 Excitation and emission spectra of three phosphors with different colors
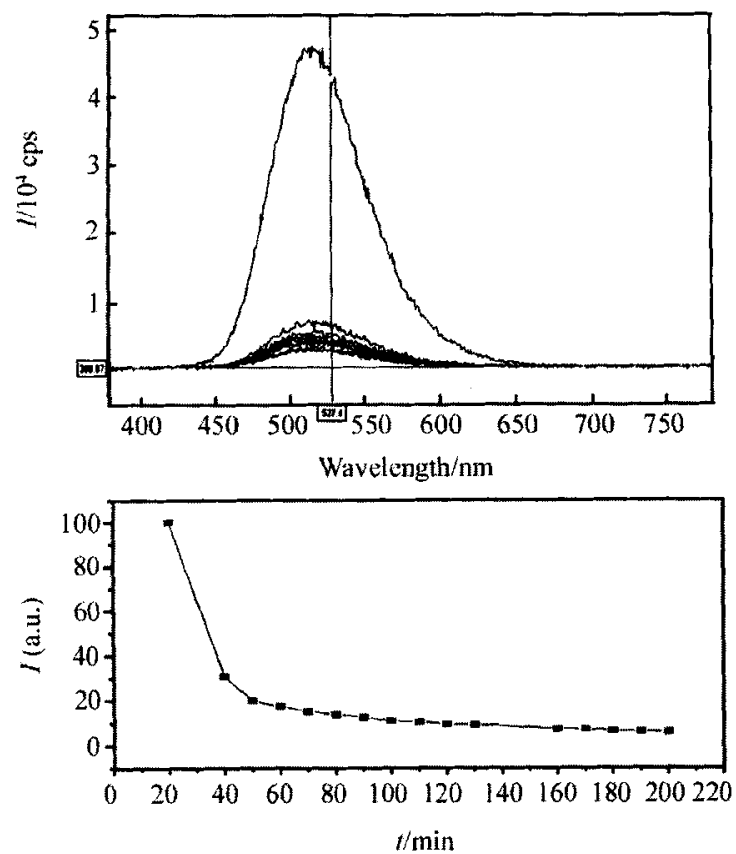

Fig.4 Emission spectra of $\mathrm{SrAl}_{2} \mathrm{O}_{4}: \mathrm{Eu}$, Dy at different time and its decay curve (Each point on the decay curve represents one curve on emission spectra) of same wave, instead of by the light with shorter wave.

\section{Conclusions}

1. The temperature of pre-polymerization should be not higher than $70^{\circ} \mathrm{C}$. By controlling pre-polymerization, it is possible to achieve the PMMA successfully. The luminescent particles in the PMMA with longpersistent phosphors made in this way would distribute uniformly .

2. The bending stress of the PMMA with longpersistent phosphors is related to the particles' distribution density in the organic glass: the bending stress is descended as the increasing of the phosphors' content.

3. The PMMA with long-persistent phosphors of the same color can excite each other due to the solid syntonic absorbability. The peak of wavelength spectra of the phosphors will not move with time.

\section{References:}

[1] Matsuzawa T, Aoki Y, Takeuchi N, et al. A new long phosphorescent phosphor with high brightness $\mathrm{SrAl}_{2} \mathrm{O}_{4}$ : $\mathrm{Eu}^{2+}, \mathrm{Dy}^{3+}[\mathrm{J}]$. J. Electrochem. Soc (in Jap.), 1996, $143(8): 2670$.

[2] Yen W, Shionoya S. (Chair Editor). Phosphor Handbook [M]. New York: CRC Press (English Version), 1999. 89.

[3] Jia W, Yuan H, Lu L. Phosphorescent dynamics in $\mathrm{SrAl}_{2} \mathrm{O}_{4}: \mathrm{Eu}^{2+}, \mathrm{Dy}^{3+}$ single crystal fibers $[\mathrm{J}]$. Lumin., 1998, 43(67): 77 .

[4] Roych M R, Mastsuda S, Tamaki H. Long decay phosphor [P]. US: 005376303A. 1994.12.27.

[5] Chen Zhonglin, Wan Tizhi, Zhang Yuqi. Rapid synthesis of green lighting phosphors with long afterglow [J]. Lighting Engineering (in Chin.), 1999, 10(2): 35.

[6] Tang Mingdao, Li Changkuan, Gao Zhiwu, et al. The study on long persistence of $\mathrm{SrAl}_{2} \mathrm{O}_{4}: \mathrm{Eu}^{2+}[\mathrm{J}]$. Chin. J. Lumin. (in Chin.), 1995, 16(1): 51.

[7] Song Qinmei, Huang Jinfei, Wu Maojun. Study on synthesis and luminescence property of europium activated strontium aluminated $[\mathrm{J}]$. Chin. J. Lumin. (in Chin. ), 1991, 12(2): 144.

[8] Wan Farong, Long Yi. The manufacture of PMMA with long-persistent phosphors and the PMMA with long-persistent phosphors which is made in this way $[\mathrm{P}]$. China: NO. ZL01 1 40304.7, 2001.

[9] Shanhu chemistry factory. Organic Glass [M]. Shanghai: Shanghai Science and Technology Publisher (in Chin. ), 1979. 196.

[10] Fang Rongchuan. Solid Spectrum [M]. Hefei : China University of Science and Technology publisher (in Chin.), 2001. 121. 